\title{
Placenta accreta after postpartum tubal sterilisation and Novasure@ endometrial ablation
}

\author{
Nnadozie Igbokwe ${ }^{1}$, Kevin Glackin ${ }^{2}$, and Harpreet Kaur ${ }^{3}$ \\ ${ }^{1}$ Queen's University Belfast \\ ${ }^{2}$ Altnagelvin Area Hospital \\ ${ }^{3}$ Watford General Hospital
}

January 11, 2021

\begin{abstract}
A case of a 42-year-old woman, Para 3 who had an unplanned pregnancy despite bilateral tubal ligation for contraception, and Novasure (R) endometrial ablation for persistent Heavy Menstrual Bleeding (HMB). The pregnancy was complicated by missed miscarriage at 14 weeks and placenta accreta. This is an uncommon event.
\end{abstract}

\section{Hosted file}

Clinical case report.pdf available at https://authorea.com/users/388714/articles/503484placenta-accreta-after-postpartum-tubal-sterilisation-and-novasure-endometrial-ablation

\section{Hosted file}

Figure 1.pdf available at https://authorea.com/users/388714/articles/503484-placenta-accretaafter-postpartum-tubal-sterilisation-and-novasure-endometrial-ablation 